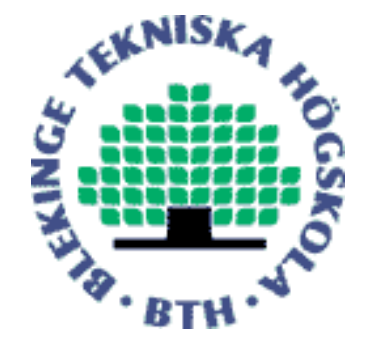

Copyright (C) 2014 IEEE.

Citation for the published paper:

Ground Moving Target Detection and Estimation By Using Dual Speed SAR Platform

Viet Thuy Vu, Mats Pettersson

RadarCon

2014 Cincinnati, Ohio

This material is posted here with permission of the IEEE. Such permission of the IEEE does not in any way imply IEEE endorsement of any of BTH's products or services Internal or personal use of this material is permitted. However, permission to reprint/republish this material for advertising or promotional purposes or for creating new collective works for resale or redistribution must be obtained from the IEEE by sending a blank email message to pubs-permissions@iee.org.

By choosing to view this document, you agree to all provisions of the copyright laws protecting it. 


\title{
Ground Moving Target Detection and Estimation By Using Dual Speed SAR Platform
}

\author{
Viet Thuy Vu and Mats I. Pettersson \\ Blekinge Institute of Technology, 37179 Karlskrona, Sweden \\ Email: viet.thuy.vu@bth.se, mats.pettersson@bth.se
}

\begin{abstract}
The paper proposes a ground moving target detection and estimation method aiming at Ultra Wide Band and -Beam (UWB) Synthetic Aperture Radar (SAR) systems. The method is developed on the moving target detection by focusing technique and requires a SAR system flying with two different speeds during the integration time. The method allows us to detect ground moving target, even hidden by clutter, and to estimate the target parameters, i.e. speed and direction of motion. The accuracy of the estimations depends strongly on the computational cost and can therefore be controlled. The proposal is tested with the simulated CARABAS data.
\end{abstract}

\section{INTRODUCTION}

Ground Moving Target Indication (GMTI) is one of the most important applications of Synthetic Aperture Radar (SAR). SAR GMTI does not only allows us to detect moving targets in an observed area but also helps us to get the images of those targets. Such information is very important for both surveillance and reconnaissance.

The moving target detection by focusing technique is one of the GMTI methods which aims at Ultra Wide Band and Beam (UWB) SAR systems [1]. The technique is based on the fact that the long integration time associated with UWB SAR strongly disperses the energy reflected from moving targets in a ground scene to different range bin. Hence, if we synthesize a large aperture from different aperture positions, the energy reflected from moving targets spreads into a large amount of grids, namely moving targets smear as either hyperbolic or elliptic curves in a SAR image, and they are therefore easily hidden by the surrounding clutter. The moving target detection by focusing technique using Normalized Relative Speed (NRS) to process the data so that the moving targets are refocused to the original shapes in the SAR image and at the same time the surrounding clutter is strongly smeared or in other words strongly suppressed.

Several researches on the technique have been carried out in order to reduce the detection time, increase the reliability of detection, and retrieve more information of the detected moving targets. Hence, the technique has been proved to work well with the frequency-domain algorithms in [2]. The technique has been integrated into a fast time-domain algorithm in [3] which allows to reduce the detection time with a factor of thousands. A combination of the technique with the spacetime processing techniques for more reliable detection has also reported in [4]. Recently, the technique has been applied to the case where the platform of a SAR system flying with two different linear flight tracks during the integration time
[5]. This allows not only detecting moving targets securely but also estimating the motion parameters of the detected moving targets accurately. However, changing direction of a SAR platform may take relatively long time compared to the integration time in certain cases, e.g. the first and the second flight tracks are identical but in opposite directions.

In this paper, we introduce a proposal of the platform motion which supports the moving target detection by focusing technique. According to this proposal, the platform moves constantly with the first speed during a part of the integration time, then accelerates to the second speed, and finally moves constantly with the second speed during the rest of the integration time. The dual speed SAR platform proposal enables both moving target detection and estimation with the moving target detection by focusing technique.

The rest of the paper is organized as follows. Section 2 presents the proposal of the platform motion in details. The procedure for moving target detection and the limits of the moving target detection by focusing technique also presented in this section. The procedures for secured moving target detection by redetection and for moving target estimation are given in Section 3. The proposal is verified by some realistic simulations based on the CARABAS parameters in section 4 . Section 5 provides the conclusions.

\section{Moving Target Detection By Focusing}

Let's consider a monostatic SAR system whose motion is assumed to be linear and with constant speeds in different time interval. Without losing the generality, the flight track (no matter what the speed of the platform is) is assumed to be parallel to $\xi$ axis as shown in Fig. 1. The SAR platform moves with the speed $v_{p l, 1}$ during the integration time $-t_{0} / 2 \leq t \leq-t_{1} / 2$. In this period, the motion of the SAR platform is expressed by

$$
\begin{aligned}
& \xi_{p l}(t)=v_{p l, 1} t \\
& \eta_{p l}(t)=0 \\
& \zeta_{p l}(t)=\zeta_{p l, 0}
\end{aligned} \text { for } \quad-t_{0} / 2 \leq t \leq-t_{1} / 2
$$

where $\zeta_{0}$ is the flight altitude. The SAR system is assumed to illuminate a ground scene where there is a moving target, e.g. a vehicle. The movement of the target in the SAR scene is also assumed to be linear and with constant speeds. The mathematical representation for the motion of the target can 


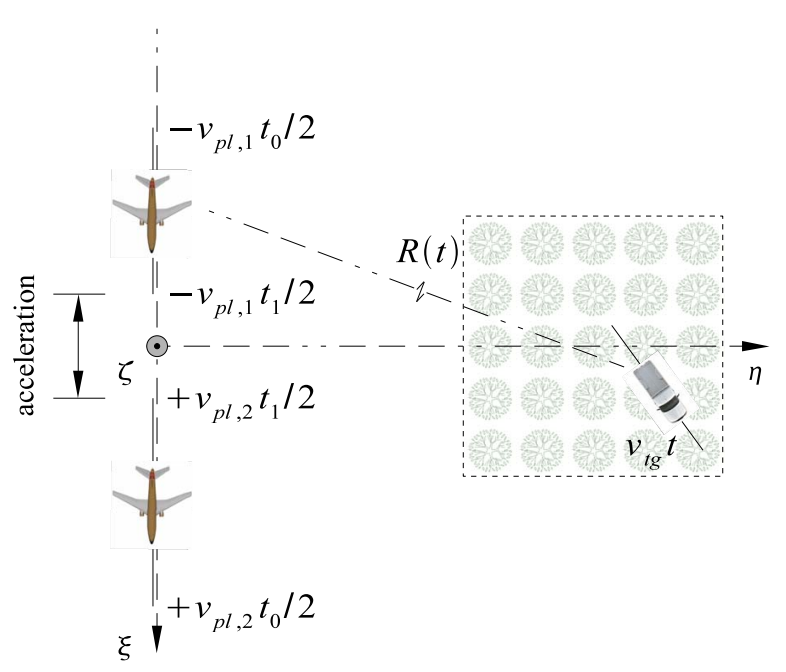

Fig. 1. SAR geometry.

be formulated by

$$
\begin{aligned}
& \xi_{t g}(t)=v_{t g, \xi} t+\xi_{t g, 0} \\
& \eta_{t g}(t)=v_{t g, \eta} t+\eta_{t g, 0} \quad \text { for } \quad-t_{0} / 2 \leq t \leq t_{0} / 2 \\
& \zeta_{t g}(t)=0
\end{aligned}
$$

where $v_{t g, \xi}$ and $v_{t g, \eta}$ are the speed components and $\left(\xi_{t g, 0}, \eta_{t g, 0}\right)$ are the coordinates of the target when $t=0$. Based on (1) and (2), the true range calculated for the SAR aperture positions during the integration time $-t_{0} / 2 \leq t \leq$ $-t_{1} / 2$ is given by

$$
\begin{aligned}
& R_{1}(t)= \\
& \quad \sqrt{\left(v_{p l, 1} t-v_{t g, \xi} t-\xi_{t g, 0}\right)^{2}+\left(v_{t g, \eta} t+\eta_{t g, 0}\right)^{2}+\zeta_{p l, 0}^{2}}
\end{aligned}
$$

If the true range history is used to reconstruct the SAR scene, the ground moving target in the SAR scene will be smeared in the SAR image under the form of either hyperbolic or elliptic curve. According to the concept of the relative speed between two objects in motion, one moving object is seen to be stationary for the other if it is assumed to move with the relative speed between them. In other words, a ground moving target can be seen to be stationary for a SAR platform if the SAR platform is assumed to move with the relative speed between them. If we call $\gamma_{1}$ is the normalized relative speed between the SAR platform moving along the first part of the flight track with the constant speed $v_{p l, 1}$ and the moving target $v_{t g}$, the relative speed of the SAR platform will be $\gamma_{1} v_{p l}$. The ground moving target is seen to be stationary at certain coordinates $\left(\xi_{1}, \eta_{1}\right)$ by the SAR platform during the integration time $-t_{0} / 2 \leq t \leq-t_{1} / 2$. In this case, the range history can also be estimated by

$$
\tilde{R}_{1}(t)=\sqrt{\left(\gamma_{1} v_{p l, 1} t-\xi_{1}\right)^{2}+\eta_{1}^{2}+\zeta_{p l, 0}^{2}}
$$

Equalizing (4) and (5) and taking the square both sides of the equation result in

$$
\begin{aligned}
\left(v_{p l, 1} t-v_{t g, \xi} t-\xi_{t g, 0}\right)^{2}+\left(v_{t g, \eta} t\right. & \left.+\eta_{t g, 0}\right)^{2} \\
& =\left(\gamma_{1} v_{p l, 1} t-\xi_{1}\right)^{2}+\eta_{1}^{2}(5)
\end{aligned}
$$

Equation (5) is a second order equation and has the form $a t^{2}+$ $b t+c=0$. However, to be assure that the condition (5) satisfies at any time $t$ during the integration time, the set of equations (6), which corresponds to the terms $t^{0}, t^{1}$ and $t^{2}$, is must be fulfilled.

$$
\left\{\begin{array}{l}
\xi_{t g, 0}^{2}+\eta_{t g, 0}^{2}-\xi_{1}^{2}-\eta_{1}^{2}=0 \\
\left(v_{p l, 1}-v_{t g, \xi}\right) \xi_{t g, 0}+v_{t g, \eta} \eta_{t g, 0}-\gamma_{1} v_{p l, 1} \xi_{1}=0 \\
\left(v_{p l, 1}-v_{t g, \xi}\right)^{2}+v_{t g, \eta}^{2}-\gamma_{1}^{2} v_{p l, 1}^{2}=0
\end{array}\right.
$$

The last equation in (6) results in NRS

$$
\gamma_{1}=\frac{\sqrt{\left(v_{p l, 1}-v_{t g, \xi}\right)^{2}+v_{t g, \eta}^{2}}}{v_{p l, 1}}
$$

We can also show that (7) can be rewritten by

$$
\gamma_{1}=\frac{\sqrt{v_{p l, 1}^{2}-2 v_{p l, 1} v_{t g} \cos \theta_{0}+v_{t g}^{2}}}{v_{p l, 1}}
$$

where $\theta_{0}$ is the angle formed by the flight track and the target trajectory as shown in Fig. 1. The first two equations in (6) give the coordinates $\left(\xi_{1}, \eta_{1}\right)$ which are not discussed in detail in this paper.

For detection, different hypotheses of NRS $\gamma_{p}$ are tested. The methods to estimate the step size between the hypotheses and the range of hypotheses are given in [4]. At a hypothesis $\gamma_{p} \approx \gamma_{1}$, the moving target is strongly focused in the SAR image whereas strong smearing occurs to the clutter. If the intensity of the focused moving target is higher than the one of the smeared clutter and if $\Lambda$ is a appropriate detection threshold, the moving target will be detected.

However, we cannot distinguish between the moving target and the clutter if a target moves in the following direction [6]

$$
\theta_{0}=\operatorname{Arccos}\left(\frac{v_{t g}}{2 v_{p l, 1}}\right)
$$

In this case, $\gamma_{1}=1$ and the SAR is therefore blind with that moving target. Fortunately, this technical issue can be solved by redetection which is presented in the next section.

\section{REDETECTION AND ESTIMATION}

During the integration time $-t_{1} / 2 \leq t \leq t_{1} / 2$ or along the second part of the flight track, the platform accelerates from increases $v_{p l, 1}$ to $v_{p l, 2}$. After accelerating, the platform moves with a constant speed $v_{p l, 2}$ during the integration time $t_{1} / 2 \leq t \leq t_{0} / 2$. The expression of the motion of the SAR platform during this integration time is given by

$$
\begin{aligned}
& \xi_{p l}(t)=v_{p l, 2} t \\
& \eta_{p l}(t)=0 \\
& \zeta_{p l}(t)=\zeta_{p l, 0}
\end{aligned} \quad \text { for } \quad t_{1} / 2 \leq t \leq t_{0} / 2
$$


If we call $\gamma_{2}$ is the normalized relative speed between the SAR platform moving along the third part of the flight track with the constant speed $v_{p l, 2}$ and the moving target $v_{t g}$, we can find $\gamma_{2}$ and the corresponding coordinates $\left(\xi_{2}, \eta_{2}\right)$ in the similar way presented in section 2 . The mathematical representation of NRS is shown to be

$$
\gamma_{2}=\frac{\sqrt{v_{p l, 2}^{2}-2 v_{p l, 2} v_{t g} \cos \theta_{0}+v_{t g}^{2}}}{v_{p l, 2}}
$$

If we apply the Taylor expansion up to the first order for (8) and (11), we can find the approximate relationship between $\gamma_{1}$ and $\gamma_{2}$ as

$$
\gamma_{2} \approx 1-\frac{v_{p l, 1}}{v_{p l, 2}}\left(1-\gamma_{1}\right)
$$

This approximate relationship (12) allows us to limit the range of hypotheses $\gamma_{p}$ in the redetection stage.

For redetection, the same procedure in the detection stage is applied. This means that we also need to test with different hypotheses of NRS $\gamma_{p}$ in a range limited by (12) needs to be tested and use a detection threshold $\Lambda$ for the moving target redetection.

We can see that, if the platform moves with the speed $v_{p l, 1}$ and if $\theta_{0}$ is given by (9), i.e. $\gamma_{1}=1$, the SAR system will be blind with the considered moving target. However, if the speed to the platform changes to $v_{p l, 2}$ the SAR system will not be blind the target, i.e. $\gamma_{2} \neq 1$. Hence, the change in speed of the platform during the integration time allows us to avoid this technical issue. In other words, we can definitely distinguish between any moving target and clutter with this proposal in order to detect the moving target.

From (8) and (11), we can derive the speed of the target $v_{t g}$ and the angle formed by the flight track and the target trajectory $\theta_{0}$ as follows

$$
v_{t g}=\sqrt{v_{p l, 1} v_{p l, 2}\left(1-\frac{\gamma_{2}^{2} v_{p l, 2}-\gamma_{1}^{2} v_{p l, 1}}{v_{p l, 2}-v_{p l, 1}}\right)}
$$

and

$$
\begin{aligned}
\theta_{0} & =\arccos \left[\frac{v_{p l, 1}^{2}\left(1-\gamma_{1}^{2}\right)+v_{t g}^{2}}{2 v_{p l, 1} v_{t g}}\right] \\
& =\arccos \left[\frac{v_{p l, 2}^{2}\left(1-\gamma_{2}^{2}\right)+v_{t g}^{2}}{2 v_{p l, 2} v_{t g}}\right]
\end{aligned}
$$

With $\gamma_{1}$ and $\gamma_{2}$ are approximately retrieved in the detection and redetection stages, we can easily estimate the speed of the target $v_{t g}$ and its motion of direction $\theta_{0}$.

\section{Proposal Validity}

In this section, we present some simulation results to verify the proposals and the derived equations given in Section II and Section III. In the simulations, a geometry similar to the one given in Fig. 1 is utilized. The data collected along the first part of the flight track is used for detection and the third part for redetection and estimation. The data belonging to the second part with the acceleration of the platform is only considered for forming the SAR image of the scene.
TABLE I

PARAMETERS OF THE SIMULATED PLATFORM.

\begin{tabular}{|c||c|}
\hline \multicolumn{1}{|c||}{ Parameter } & Value \\
\hline \hline The highest frequency processed & $82 \mathrm{MHz}$ \\
\hline The lowest frequency processed & $22 \mathrm{MHz}$ \\
\hline The first speed of platform & $126 \mathrm{~m} / \mathrm{s}$ \\
\hline Flight altitude & $3700 \mathrm{~m}$ \\
\hline Minimum range to the aim point & $7150 \mathrm{~m}$ \\
\hline Pulse Repetition Frequency (PRF) & 137 \\
\hline Aperture step & $0.9375 \mathrm{~m}$ \\
\hline \hline Acceleration & $1 \mathrm{~m} / \mathrm{s}^{2}$ \\
\hline The second speed of platform & $151 \mathrm{~m} / \mathrm{s}$ \\
\hline Integration time & $75 \mathrm{~s}$ \\
\hline Number of aperture positions & 10166 \\
\hline
\end{tabular}

\section{A. Arrangement}

The simulated SAR system is mainly based only on the parameters of CARABAS in reality [2] and they are summarized in the upper part of Table I. Other parameters required by the simulations are given in the lower part. As shown in Table I, after the acceleration with $1 \mathrm{~m} / \mathrm{s}^{2}$, the speed of the platform is increased by $20 \%$. The total integration time includes the integration times of the first part of the flight track with the constant speed $v_{p l, 1}$, the second part with the constant acceleration $a_{p l}$, and the third part with the constant speed $v_{p l, 2}$. The integration time (75 s) corresponds to 10166 aperture positions.

The simulated SAR scene consists of an arbitrary moving target which is the subject to be detected and estimated and covered by clutter. The moving target is a point-like scatterer and its Radar Cross Section (RCS) is normalized to 1 . The speed of the target is chosen by $10 \mathrm{~m} / \mathrm{s}$ and the direction of motion is $15^{\circ}$ with respect to the $\xi$ axis. The clutter is a set of point-like scatterers and equally spaced in the area where the moving target presents. The RCS of the clutter are given by the pseudorandom values drawn from the standard uniform distribution on the open interval $(0,1)$ and scaled with a factor of $1 / 2$.

Fig. 2 shows the SAR image of the simulated scene formed by the GBP algorithm using the true ranges. The data belonging to the second part of the flight track, i.e. the data collected during the SAR platform acceleration, is considered in the SAR image formation. The area of the SAR scene to be reconstructed is $512 \mathrm{~m} \times 512 \mathrm{~m}$ with the azimuth and range samples are $1 \mathrm{~m} \times 1 \mathrm{~m}$. Fig. 2 shows this SAR image where the middle part of the SAR image is of interest. In this area, the clutter is well focused and appears everywhere in the middle part of the SAR image of the SAR image. At the same time, we cannot observe the moving target of interest since it is supposed to be blurred in the SAR image and totally obscured by the clutter.

In this simulation, we can also see the flexibility of GBP as it can still synthesize well the full aperture regardless of the complex motion of the SAR platform, i.e. acceleration, during the data collection. 


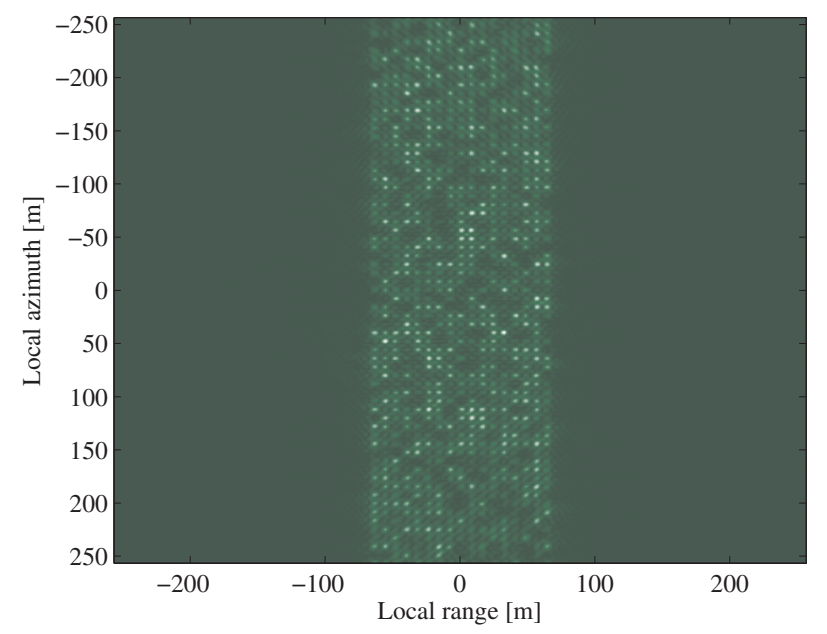

Fig. 2. SAR image of the simulated ground scene. The image is reconstructed with the second flight track data.

\section{B. Detection}

For detection, different hypotheses of $\gamma_{1}$ are tested on the data collected along the first part of the flight track. An optimum quantization step size between hypotheses is selected by $\Delta \gamma=5 \cdot 10^{-3}$. This selection corresponds to the threshold of $h_{\text {lim }}=3 \mathrm{~dB}$ allowing maximum loss of $3 \mathrm{~dB}$ from the peak intensity of the moving target in the SAR image. If we need to detect moving targets whose speed is less than $10 \%$ of the platform speed, the range of hypotheses will be in the interval $\gamma_{p} \in[0.9,1.1]$. Hence, we have totally 41 hypotheses of $\gamma_{1}$. The area of interest or the observed area in this simulation is the middle part of the SAR image. In reality, areas of interest can also be very large, e.g. forest where moving targets can be hidden by thick foliage, a water area where vessels are prevented to go into, or a long road where over speed vehicles need to be indicated.

Fig. 3 shows the detection result which is presented by the relationship between $\gamma_{p}$ and the retrieved peak intensities in the area of interest (continuous curve). As observed from the 2-D graph, two peak intensities occur at the hypotheses $\gamma_{p}=$ 0.925 and $\gamma_{p}=1.000$. The former is supposed to correspond to the moving target which need to be detected and the latter should be associated with the clutter. If we set a detection threshold of, for example $\Lambda=3 \mathrm{~dB}$ and apply it for the found peak intensities, the presence of a moving with NRS of 0.925 in the area of interest will be indicated. In other words, the moving target is detected and the estimated NRS for the detected target is about $\gamma_{1} \approx 0.925$.

\section{Redetection}

With the value of $\gamma_{1}$ retrieved in the detection stage, we can sparsely estimate $\gamma_{2}$ using (11) as $\gamma_{2} \approx 0.9375$. With this approximation, it is sufficient to examine $\gamma_{2}$ in the short range of hypotheses for the redetection. Based on the hypotheses in the detection stage, the range of hypotheses for the redetection

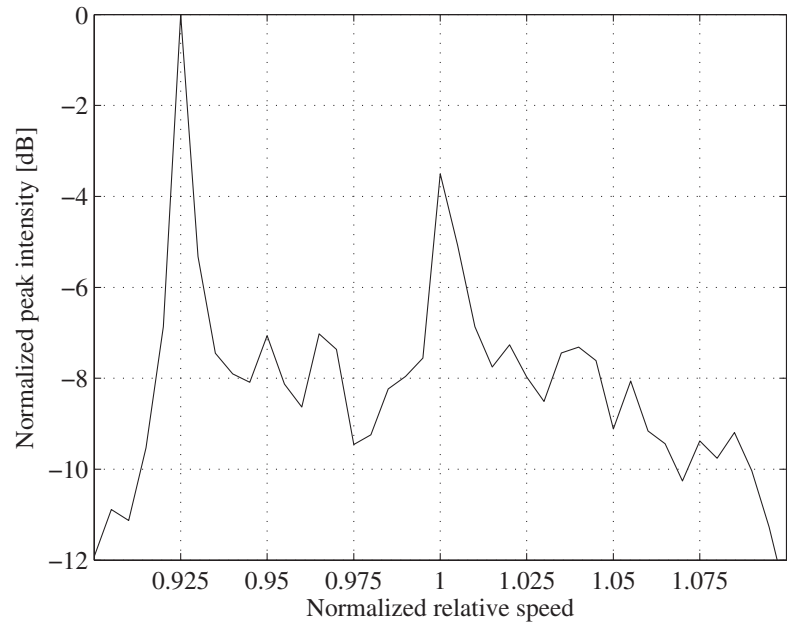

Fig. 3. Detection result with two peak intensities associated with the hypotheses $\gamma_{p}=0.925$ and $\gamma_{p}=1.000$.

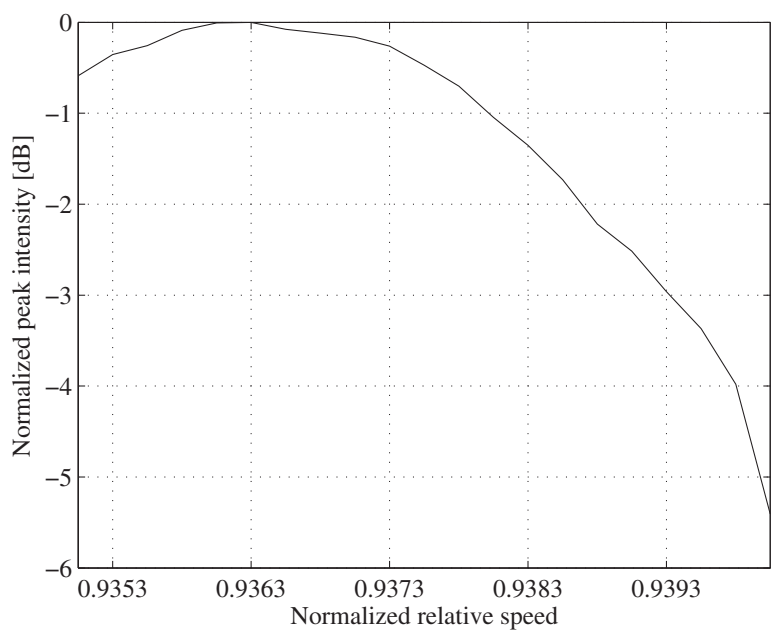

Fig. 4. Redetection result with the peak intensity associated with the hypothesis $\gamma_{p}=0.9363$.

can be limited by $\gamma_{p} \in[0.935,0.940]$. It is obvious that this range of hypotheses is narrower than the one for the detection stage 40 times. We can reduce the step between the tested hypotheses to get a better estimation of $\gamma_{2}$ which can be used directly in the estimation stage. In this illustration, we select $\Delta \gamma=1 \cdot 10^{-4}$. This means that the step size in the redetection stage is smaller than the step one in the detection stage 50 times. The number of hypotheses is therefore 51. Based on the 2-D graph of the relationship between $\gamma_{p}$ and the peak intensities shown in Fig. 4, we can securely detect the moving target and retrieve $\gamma_{2} \approx 0.9363$.

\section{Estimation}

For accurate estimation, it is necessary to test the focusing of the moving target by smaller step sizes between hypotheses surrounding $\gamma_{1}$ retrieved in the detection stage. This allows us 


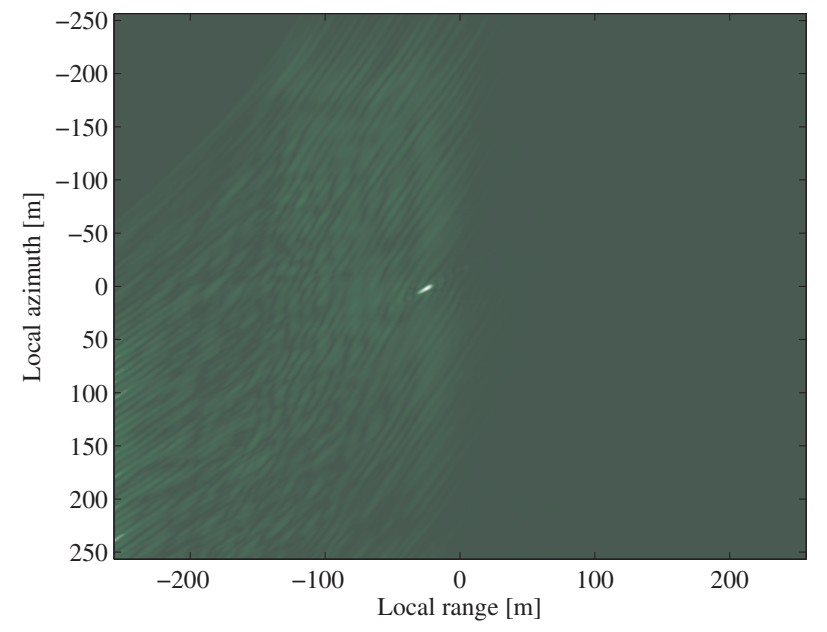

Fig. 5. SAR image reconstructed with the data collected along the first part of the flight track and with $\gamma_{p}=0.9236$.

to get the more accurate estimation of $\gamma_{1}$. In this illustration, the range of hypotheses for the estimation of $\gamma_{1}$ can be defined by $\gamma_{p} \in[0.9225,0.9275]$ and the step between the hypotheses is similar to the one used in the redetection stage. The result is that we obtain a better estimation of $\gamma_{1}$, i.e. $\gamma_{1}=0.9236$. Substituting these values to (13) results in an estimation for the speed of the target $\tilde{v}_{t g} \approx 9.9924 \mathrm{~m} / \mathrm{s}$ in comparison to the simulated target speed of $v_{t g}=10 \mathrm{~m} / \mathrm{s}$. The angle between the flight track and the target trajectory is calculate with (14) and given by $\theta_{0}=14.8881^{\circ}$ in comparison to the simulated target direction of motion of $15^{\circ}$. Hence, these estimations are very close to the true values of the moving target which have been used in the simulations.

From the estimations of $v_{t g}$ and $\theta_{1}$, we can also estimate the initial coordinates as well the last coordinates of the moving target during the integration time. Hence, the information of the target gives us directly $v_{t g, \xi}$ and $v_{t g, \eta}$. The coordinates $\left(\xi_{1}, \eta_{1}\right)$ can be found from SAR images with the focused moving targets. Other parameters like the estimated value of $\gamma_{1}$ are also available. The initial coordinates of the moving target $\left(\xi_{t g, 0}, \eta_{t g, 0}\right)$ can be calculated with the first two equations in the set of equations (6). The last coordinates can be retrieved from the set of equations (2).

\section{E. Moving target imaging}

The SAR image of the moving target is obtained by processing the data collected along the first part of the flight track with $\gamma_{p}=0.923$. This image is given in Fig. 5. As observed, the moving target is now refocused to a point target and appears in the middle of the SAR scene while the clutter is migrated and suppressed significantly.

We can also form the SAR image of the moving target by processing the data collected along the third part of the flight track with $\gamma_{p}=0.936$ as shown in Fig. 6. The same effects with the moving target and the clutter can be observed

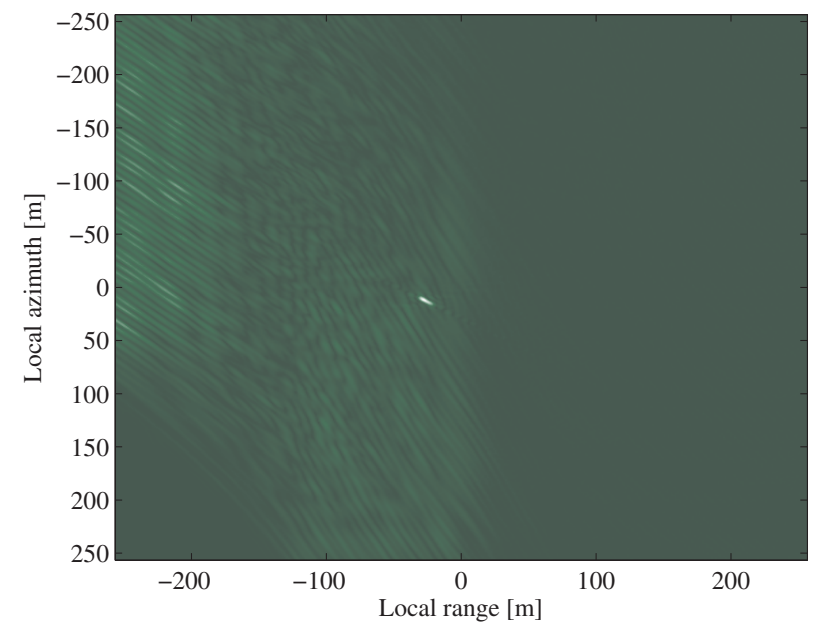

Fig. 6. SAR image reconstructed with the data collected along the third part of the flight track and with $\gamma_{p}=0.9363$.

from Fig. 6. However, the moving target and the cluster look differently in the SAR images due to the data collected at different parts of the flight track is used for reconstructing the SAR images.

\section{CONCLusions}

In this paper, we have proposed a ground moving target detection and estimation method aiming at UWB SAR systems. The method overcomes some shortcomings of the current version of the moving target detection by focusing technique. To ensure the method work well, a SAR platform needs to flight with two different speeds during the integration time. With the proposed method, we have a higher possibility to detect moving targets in a ground scene or in other words a more reliable detection. The method allows estimating all parameters of a detected moving target including speed, direction of motion, initial coordinates and last coordinates.

\section{REFERENCES}

[1] V.T. Vu, T.K. Sjögren, M.I. Pettersson, A. Gustavsson, and L.M.H Ulander, "Moving targets detection by focusing in UWB SAR - Theory and experimental results," IEEE Trans. Geosci. Remote Sensing, vol. 48, no. 10 , pp. 3799-3815, 2010.

[2] V.T. Vu, T.K. Sjögren and M.I. Pettersson, "Moving target detection by focusing for frequency domain algorithms in UWB low frequency SAR," in Proc. IEEE IGARSS'2008, Boston, MA, Jul. 2008, pp. IV-1284-IV1287.

[3] V.T. Vu, T.K. Sjögren and M.I. Pettersson, "Fast detection of moving targets by focusing in ultrawideband SAR," in Proc. IEEE RadarCon'2009, Pasadena, CA, May 2009, pp. 1-5.

[4] V.T. Vu, T.K. Sjögren and M.I. Pettersson, "Integrating space-time processing into time-domain backprojection process to detect and image moving objects," in Proc. IEEE IGARSS'2010, Honolulu, HI, Jul. 2010, pp. 4106-4109.

[5] V.T. Vu, T.K. Sjögren and M.I. Pettersson, "Ground moving target detection and estimation with different SAR linear flight tracks," in Proc. IEEE IGARSS'2013, Melbourne, Australia, Jul. 2013, pp. 2031-2034.

[6] A. Gustavsson, L.M.H. Ulander, B.H. Flood, P.-O. Frölind, H. Hellsten, T. Jonsson, B. Larsson, and G. Stenstrom, "Development and operation of an airborne VHF SAR system-lessons learned," in Proc. IEEE IGARSS'98, vol. 1, Seattle, WA, Jul. 1998, pp. 458-462. 